\title{
Regulação hormonal da ingestão alimentar: um breve relato
}

\section{Hormonal regulation of food intake: $A$ brief review}

Alex Souto Maior ${ }^{1,2}$

\begin{abstract}
RESUMO
O objetivo deste trabalho de revisão foi dissertar sobre a regulação hormonal da ingestão alimentar e do peso corporal. A ingestão de macronutrientes, o gasto energético e o balanço entre a síntese de hormônios que aumentam a atividade dos neurônios orexígenos e anorexígenos são fatores determinantes na regulação da ingestão alimentar e no controle do peso corporal. Os hormônios leptina, insulina e o peptídeo YY são grandes potenciadores da atividade dos neurônios anorexígenos POMC/CART. Por outro lado, a ação da grelina aumenta a atividade dos neurônios orexígenos NPY/AgRP. Estes hormônios sintetizados perifericamente atravessam a barreira-hemato-encefálica e se ligam aos seus receptores no núcleo arqueado hipotalâmico com o objetivo de regular a ingestão alimentar e controlar o balanço energético. Estes mecanismos revelam a complexidade do controle do balanço energético e todos os centros envolvidos na busca pela homeostase energética.
\end{abstract}

Palavras-chave: Hormônios. Neurônios Orexígenos e Anorexígenos. Ingestão Alimentar. Neurônios/ classificação.

\section{Introdução}

A busca pela homeostasia da ingestão alimentar ocorre a partir de um conjunto de reações químicas, hormonais e centrais no organismo vivo. ${ }^{1}$ Estas reações promovem o crescimento e o desenvolvimento do organismo através da ingestão de macronutrientes, do gasto energético, da termogênese dos alimentos e do balanço entre a síntese de hormônios que aumentam a atividade dos neurônios orexígenos e anorexígenos. ${ }^{2,3,4}$

Durante as refeições, o trato gastrointestinal, equipado com quimiorreceptores e mecanorreceptores especializados, monitoram a quantidade de alimentos ingeridos e a qualidade do conteúdo dos nutrientes. ${ }^{1,2}$ No estado pós-prandial os nutrientes passam pelo processo digestivo, atravessam a parede intestinal e entram na circulação. ${ }^{1,2}$ Este monitoramento informa ao hipotálamo, por meio das fibras aferentes vagais, os sinais de saciedade e, consequentemente o controle do apetite pré-absortivo. ${ }^{1,2,3}$ Esta alça de feedback mostra a interação entre hormônios e as vias hipotalâmicas na regulação da ingestão alimentar e no armazenamento e utilização das reservas energéticas. ${ }^{1,2}$

$\mathrm{O}$ desequilíbrio do balanço energético e o aumento dos tecidos adiposos parecem contribuir com a
1- Doutor em fisiologia - Universidade Federal do Rio de Janeiro - Instituto de Biofísica Carlos Chagas Filho (UFRJ/IBCCF).

2- Professor do Departamento de Fisiologia do exercício - Universidade Castelo Branco (UCB/RJ).
Correspondência: Prof.Dr. Alex Souto Maior Universidade Castelo Branco Avenida Santa Cruz, 1631 - Realengo 21710-250 - Rio de Janeiro/RJ - Brasil. e-mail: alex.bioengenharia @ gmail.com

Artigo recebido em 19/12/2011 Aprovado para publicação em 25/06/2012 
resistência da ligação da leptina aos seus receptores hipotalâmicos, fato que inibe a atividade dos neuropéptidos anorexígenos. ${ }^{4,5}$ Além disso, este desequilíbrio pode promover resistência à insulina, diminuição do gasto energético e lesão endotelial. ${ }^{4,5}$ Por outro lado, a atividade dos neuropeptídeos orexígenos está relacionada à síntese da grelina que promove o balanço energético positivo. ${ }^{5,6} \mathrm{Em}$ suma, o objetivo deste artigo de revisão foi dissertar sobre o papel da regulação hormonal na ingestão alimentar e no controle do peso corporal a partir de busca bibliográfica em banco de dados como: MedLine e PubMed.

\section{Fatores neurais e regulação da ingestão alimentar}

O hipotálamo é a região essencial do sistema nervoso central que regula a ingestão alimentar e o balanço energético. ${ }^{1,5,6}$ No hipotálamo existem duas importantes vias neurais (lateral e ventromedial) que regulam o comportamento metabólico a partir da atividade endócrina, autonômica e do controle do dispêndido e armazenamento de energia de forma coordenada. ${ }^{6}$ Em modelos animais e humanos, o centro hipotalâmico lateral é responsável pela regulação da alimentação e o centro hipotalâmico ventromedial é responsável pela saciedade. ${ }^{7,8}$ É importante comentar que lesões hipotalâmicas (região lateral) promovem redução da ingestão alimentar e apresentam quadro de afagia. ${ }^{7,8}$ Por outro lado, lesões no núcleo arqueado, induzidas pela utilização de glutamato monossódico, por exemplo, provocam um quadro de obesidade, hiperfagia e diminuição da atividade simpática. ${ }^{1,6,9}$ Lesões no centro hipotalâmico ventromedial mostram aumento do peso corporal e hipogonadismo pela diminuição da saciedade. ${ }^{6,7,10}$

O hipotálamo apresenta dois grandes grupos de neuropeptídeos (orexígenos e anorexígenos) envolvidos na regulação da ingestão alimentar. Os neuropeptídeos orexígenos (que estimulam a ingestão alimentar) são o neuropeptídeo Y (NPY) e o peptídeo relacionado à Agouti (AgRP) (um antagonista dos receptores de melanocortina). ${ }^{10}$ Os neuropeptídeos anorexígenos (que reduzem a ingestão alimentar) são o próopiomelanocortina (POMC) e o transcrito relacionado à cocaína e à anfetamina (CART) ${ }^{10}$

A ação dos hormônios que potencializam o efeito anorexígeno no sistema nervoso central (SNC) ocorre a partir da ativação de neurônios no núcleo arqueado hipotalâmico que expressam a POMC e promovem a síntese do hormônio alfa-melanócito estimulador
$(\alpha-\mathrm{MSH})$. Este se liga aos receptores melanocortina 4 promovendo a redução da ingestão alimentar., ${ }^{5,10,11}$ O aumento da expressão do CART no núcleo arqueado também é responsável pela inibição da ingestão alimentar. ${ }^{10,11}$ Por outro lado, os hormônios que potencializam o efeito orexígenos contribuem com o balanço energético positivo por aumentar a expressão dos neurônios NPY/AgRP que antagonizam os efeitos da POMC e do CART. ${ }^{5,10,11}$ Este antagonismo está relacionado à inibição dos neurônios do núcleo paraventricular que expressam o neurotransmissor corticotrofina (função anorexígena), contrariamente, a área hipotalâmica lateral expressa a orexina que exerce a função orexigênico. ${ }^{10,11}$ Assim, há aumento da resposta excitatória para os neurônios NPY/AgRP e inibição de os neurônios POMC/CART. ${ }^{10,11}$

\section{Interação endócrina e neural na regulação da ingestão alimentar}

É descrito na literatura que durante a ingestão alimentar ocorre o aumento da atividade dos neurônios NPY/AgRP a partir do elevação do níveis gástricos do hormônio grelina, porém após a alimentação este hormônio retorna aos níveis basais. ${ }^{10,11} \mathrm{O}$ aumento dos hormônios leptina, insulina e peptídeo YY (PYY) elevam a produção do $\alpha-\mathrm{MSH}$, consequentemente, antagonizando a atividade dos neurônios NPY/ AgRP. ${ }^{10,11}$

A leptina é um peptídeo de $16 \mathrm{kDa}$ composto por 146 aminoácidos, sintetizado e secretado principalmente pelas células do tecido adiposo branco. ${ }^{1,3} \mathrm{O}$ gene $o b$ da leptina localiza-se no cromossomo 7q31 com baixa expressão no epitélio gástrico e na placenta. ${ }^{1}$ Em humanos, o ritmo circadiano deste hormônio apresenta pico de secreção durante a noite e nas primeiras horas da manhã, sendo sua meia-vida plasmática de 30 minutos. ${ }^{1,3}$

A leptina apresenta receptores $\mathrm{ObRb}$ de cadeia longa (maior quantidade de aminoácidos) com maior expressão no núcleo de arqueado do hipotálamo, mais precisamente no hipotálamo ventromedial e dorsomedial, na área hipotalâmica lateral e na área pré-óptica medial. ${ }^{1,3,5,7}$ A leptina tem a função de aumentar a expressão dos neurônios anorexígenos POMC/CART, consequentemente, antagoniza a atividade dos neurônios orexígenos NPY/AgRP. ${ }^{3,10,11}$ Estas evidências fundamentam que os efeitos da leptina sobre o balanço energético são mediados por neurônios desta região que suprimem a ingestão alimentar e controlam o gasto energético. 
A ligação da leptina repercute na dimerização e na mudança conformacional do receptor ObRb que induz a atividade catalítica de uma proteína citosólica chamada de Janus quinase -2 (JAK-2) promovendo o recrutamento de moléculas da família de transdutores de sinal e ativadores de transcrição (STAT 3). A Leptina estimula a atividade da STAT 3 nos neurônios da POMC pelo recrutamento das histonas acetilases, consequentemente, o STAT 3 inibe a expressão do RNAm dos neurônios AgRP, e possivelmente do NPY, pelo recrutamento das histonas deacetilases., ${ }^{3,11} \mathrm{O}$ aumento da atividade dos neurônios da POMC induz ao aumento da expressão dos neurônios da CART (figura 1). Este mecanismo hipotalâmico obtêm informações referentes à quantidade de energia armazenada no tecido adiposo e a resposta termogênica nos terminais sinápticos..$^{3,11}$

Estas afirmações são comprovadas a partir de estudos experimentais que administraram leptina nos ventrículos cerebrais e observaram aumento da expressão do fator de transcrição STAT 3 no núcleo arqueado hipotalâmico. ${ }^{3,10}$ Estas evidências confirmam a participação da leptina sobre o controle da saciedade. Por outro lado, a obesidade parece favorecer a resistência da leptina em se acoplar ao seu receptor hipotalâmico possivelmente por anormalidades na secreção de leptina, déficits no transporte de leptina na barreira hemato-encefálica e reduzida sinalização hipotalâmica de leptina. ${ }^{1,3,10,12}$ Em modelos animais obesos é visto que a administração de leptina central não promove alteração da atividade do STAT3, consequentemente, redução do controle da saciedade. ${ }^{12}$

Como mencionado anteriormente a leptina promove a expressão da POMC que sintetiza o $\alpha-\mathrm{MSH}$. Este se liga aos receptores de melanocortina ( $\mathrm{MCr} 3$ e 4) regulando o peso corporal e controlando a ingestão calórica. ${ }^{3,6} \mathrm{Os} \mathrm{MCr} 3$ são expresso no SNC, na placenta, no intestino, no timo e nos adipócitos e sua ligação ao $\alpha-\mathrm{MSH}$, principalmente nos adipócitos, representam importante função no gasto energético e na lipólise. ${ }^{3,6}$ Os MCr 4 são abundantemente expressos no hipotálamo e a sua ligação ao $\alpha$-MSH tem uma importante função no controle da saciedade. ${ }^{3}$

A síntese da leptina e a expressão dos $\mathrm{MCr} 4$ tem um importante papel inibitório da proteína quinase ativadora mitogênica (AMPK) no hipotálamo paraventricular ${ }^{3,11}$. Estudos experimentais observaram que animais tratados com leptina apresentaram significa-

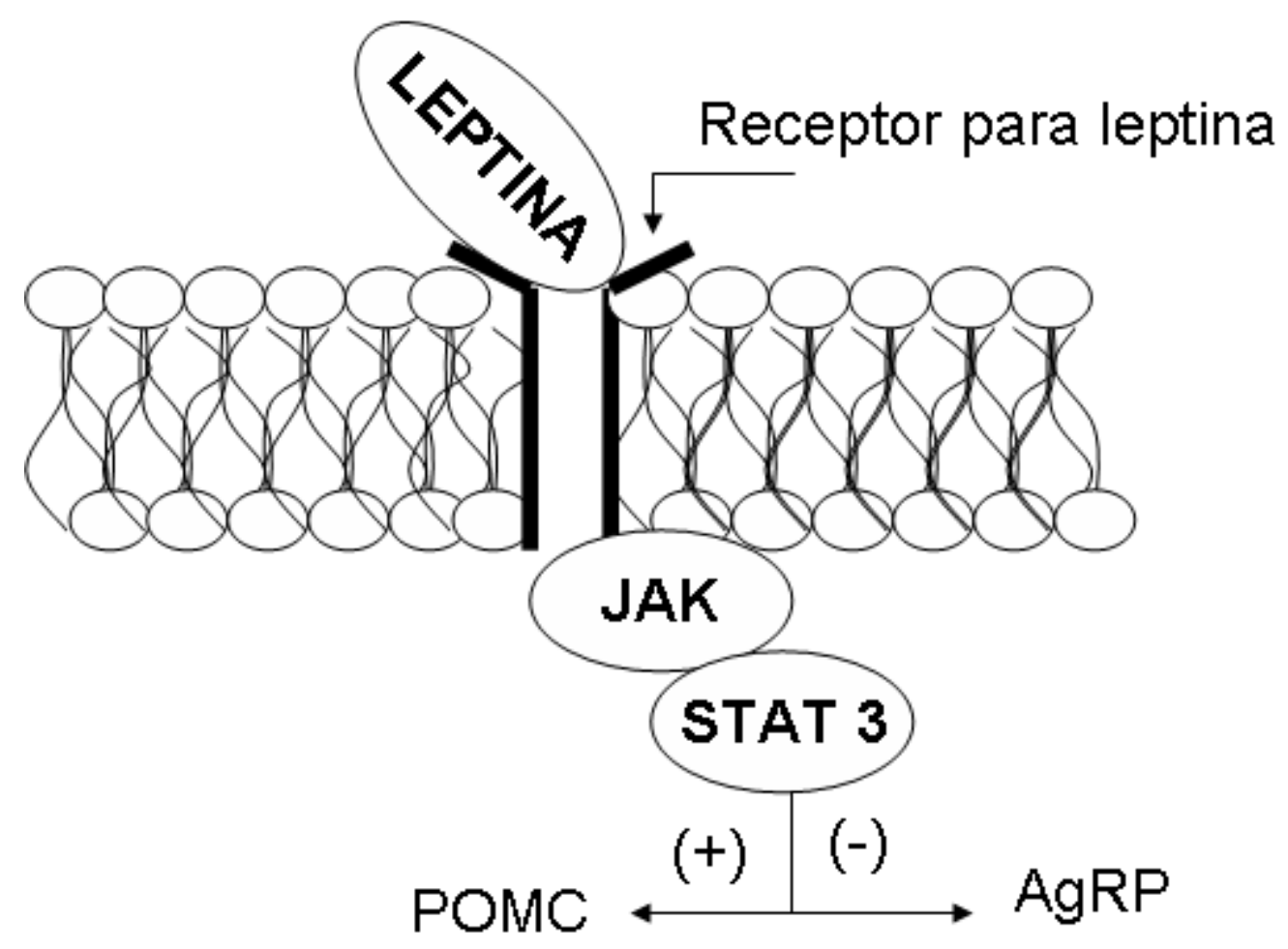

Figura 1. Diagrama esquemático do neurocircuito hipotalâmico revela a ligação da leptina ao seu receptor que favorece o sinal de transdução ao sistema JAK/STAT3 pelo aumento da expressão da POMC e inibição do AgRP. 
tiva supressão da atividade da AMPK no hipotálamo. ${ }^{13}$ Contrariamente, a AMPK tem papel importante na regulação da oxidação de ácidos graxos no músculo esquelético. ${ }^{13,14}$ A AMPK é ativada quando a razão celular de AMP/ATP aumenta após diminuição dos níveis de ATP, consequentemente, repercute na oxidação dos ácidos graxos para obtenção de ATP e inibição das vias metabólicas para consumo de ATP. ${ }^{13,14}$

É importante comentar que a ingestão alimentar hipercalórica associada à redução do gasto energético promove o aumento significativo das concentrações séricas de leptina. Contudo, na busca pela homeostase energética ocorre o aumento significativo da síntese de leptina pelos adipócitos com intuito de promove a lipólise e inibir a lipogênese. ${ }^{12,15}$ Desta forma, é considerado um potente agente de proteção contra a adipotoxicidade provocada pelo excesso de triacilgliceróis no citossol em células que não são adipócitos. ${ }^{15}$

A grelina é um hormônio orexígeno composto de 28 aminoácidos com uma modificação octanóica no seu grupo hidroxil sobre a serina 3 , sendo este fator essencial para estimular a síntese do hormônio do crescimento $(\mathrm{GH})$ a partir da sua ligação aos receptores secretagogo de GH do tipo 1 (GHS-R). ${ }^{1,16}$ Outro função relevante da grelina ao ligar-se com GHS-R do tipo 1 é a estimulação da secreção ácida e o esvaziamento gástrico em humanos. ${ }^{18} \mathrm{Em}$ algumas patologias como anorexia nervosa este hormônio apresenta níveis elevados, contrariamente, na obesidade os níveis plasmáticos de grelina estão reduzidos. ${ }^{16,17,18}$

A síntese deste hormônio ocorre primeiramente na mucosa oxíntica do estômago sendo secretada em células especializadas enterocromafins no trato gastrointestinal. ${ }^{16} \mathrm{O}$ ciclo circadiano em humanos da grelina apresenta uma fase de variação diurna de acordo com o nível plasmático de leptina. ${ }^{17}$ Assim, os picos de secreção de grelina têm sido encontrados antes de cada refeição, com queda significativa imediatamente após a ingestão alimentar. ${ }^{1,17,18}$ Os níveis de concentrações plasmáticas da grelina podem variar de acordo com o tipo de macronutriente contido na ingestão alimentar, ou seja, refeições ricas em proteína animal e lipídeos apresenta níveis plasmáticos de grelina significativamente elevados em relação a refeições ricas carboidratos. ${ }^{1,19}$

Embora a grelina seja sintetizada perifericamente, ela apresenta receptores específicos no sistema nervoso central (SNC). Este hormônio se liga ao seu receptor no núcleo arqueado hipotalâmico e aumenta a atividade dos neurônios NPY/AgRP e, antagonicamente, inibe os neurônios da POMC a partir da liberação pré-sináptica do ácido gama-aminobutírico (GABA). ${ }^{1,5,8,20}$ Em modelos animais a administração da grelina centralmente estimula os neurônios orexígenos, principalmente os neurônios NPY, para regulação da homeostase energética. ${ }^{21,22}$ Esta infusão de grelina pode aumentar a ingestão alimentar em 30\% por suprimir a saciedade pós-prandial. ${ }^{22}$

No período pós-prandial, o trato gastrointestinal possui células L que secretam o peptídeo YY (PYY). Este peptídeo regula a motilidade intestinal e aumenta a saciedade a partir da interação com o sistema melanocortina na área tegumentar ventral e no striatum ventral. ${ }^{23,24} \mathrm{O}$ PYY se liga ao receptor pré-sináptico inibitório Y2 que aumenta a atividade dos neurônios anorexígenos POMC/CART e inibe a atividade dos neurônios NPY/AgRP no núcleo arqueado do hipotálamo. ${ }^{24,25}$ A secreção do PYY apresenta níveis plasmáticos elevados 1 a $2 \mathrm{~h}$ após as refeições e permanece elevado até $6 \mathrm{~h}$ pós-refeição. ${ }^{23,24}$ Este fato pode estar associado a alimentos ricos em lipídeos quando comparados com alimentos protéicos ou ricos em carboidratos. ${ }^{26}$ Por outro lado, o controle da saciedade é reduzido em indivíduos obesos pelos baixos níveis plasmáticos de PYY em jejum e pós-prandial. ${ }^{24,25,27}$ Porém, a administração venosa de PYY induz a saciedade e reduz em $30 \%$ a ingestão alimentar em pacientes obesos. $^{28}$

A insulina é um polipeptídeo com massa molecular de 5800Da produzido pelas células beta pancreáticas nas ilhotas de Langerhans. De forma semelhante à leptina, sua concentração plasmática é proporcional à adiposidade. ${ }^{29} \mathrm{O}$ aumento do tecido adiposo pode ser um determinante para desencadear a resistência à insulina a partir do aumento da atividade da proteína kinase $\mathrm{C}$ delta. ${ }^{30}$ Desta forma, a concentração de gordura corporal é um determinante para o comportamento da sensibilidade a insulina. ${ }^{29}$

A ação fisiológica da insulina é relatada na literatura em três condições específicas ${ }^{31}: 1$ ) Efeito imediato: ocorre em vários segundos após sua administração ou síntese promovendo o transporte da glicose e a fosforilação de enzimas específicas; 2) Efeito a médio-prazo: ocorre entre 5 e 60 minutos após sua administração ou síntese; 3) Efeito a longo-prazo: ocorre por vários dias após sua administração ou síntese e promove a síntese do DNA, a divisão celular e a diferenciação celular das células específicas.

Este hormônio apresenta receptores específi- 
cos no fígado, no músculo, no tecido adiposo, e alguns relatos revelam receptores também nas células vermelhas e no SNC. ${ }^{32}$ A literatura científica revela que a insulina é um potente sinalizador anorexigênico no SNC promovendo a redução da expressão dos genes hipotalâmicos que regulam a saciedade. ${ }^{33}$ No hipotálamo a insulina apresenta receptores específicos no núcleo arqueado e nas regiões dorsomedial, paraventricular e periventricular hipotalâmica. ${ }^{34}$

O receptor de insulina é derivado de um único gene no cromossomo 19 e consiste de 22 éxons separados por 21 introns. ${ }^{35}$ Estes receptores recrutam várias moléculas intracelulares envolvidas no processo de transdução. Entre elas estão os substratos protéicos dos receptores de insulina (IRS) que são fosforilados em resíduos de tirosina pelos receptores ativos de insulina. ${ }^{11}$ Este procedimento permite que o IRS expresse a enzima fosfatidilinositol-3-OH Kinase (PI(3)K) que aumenta a atividade do fosfatidilinositol-3,4,5trifosfato (PIP3) a partir do recrutamento de fosfatidilinositol-4,5-bifosfato (PIP2). ${ }^{11,36} \mathrm{~A}$ forma ativa da PIP3 sintetiza a proteína kinase dependente de fosfoinositol-3 (PDK1) que ativa a proteína kinase B (PKB), que por sua vez inibe o fator de transcrição FOXO1 (Figura 2). ${ }^{36}$ Esta inibição do FOXO1 suprime a atividade da POMC, fato associado à maior atividade da STAT3 mediado pela leptina (figura 2). ${ }^{11,36} \mathrm{Tem}$ sido descrito que a insulina segue o percurso similar ao da

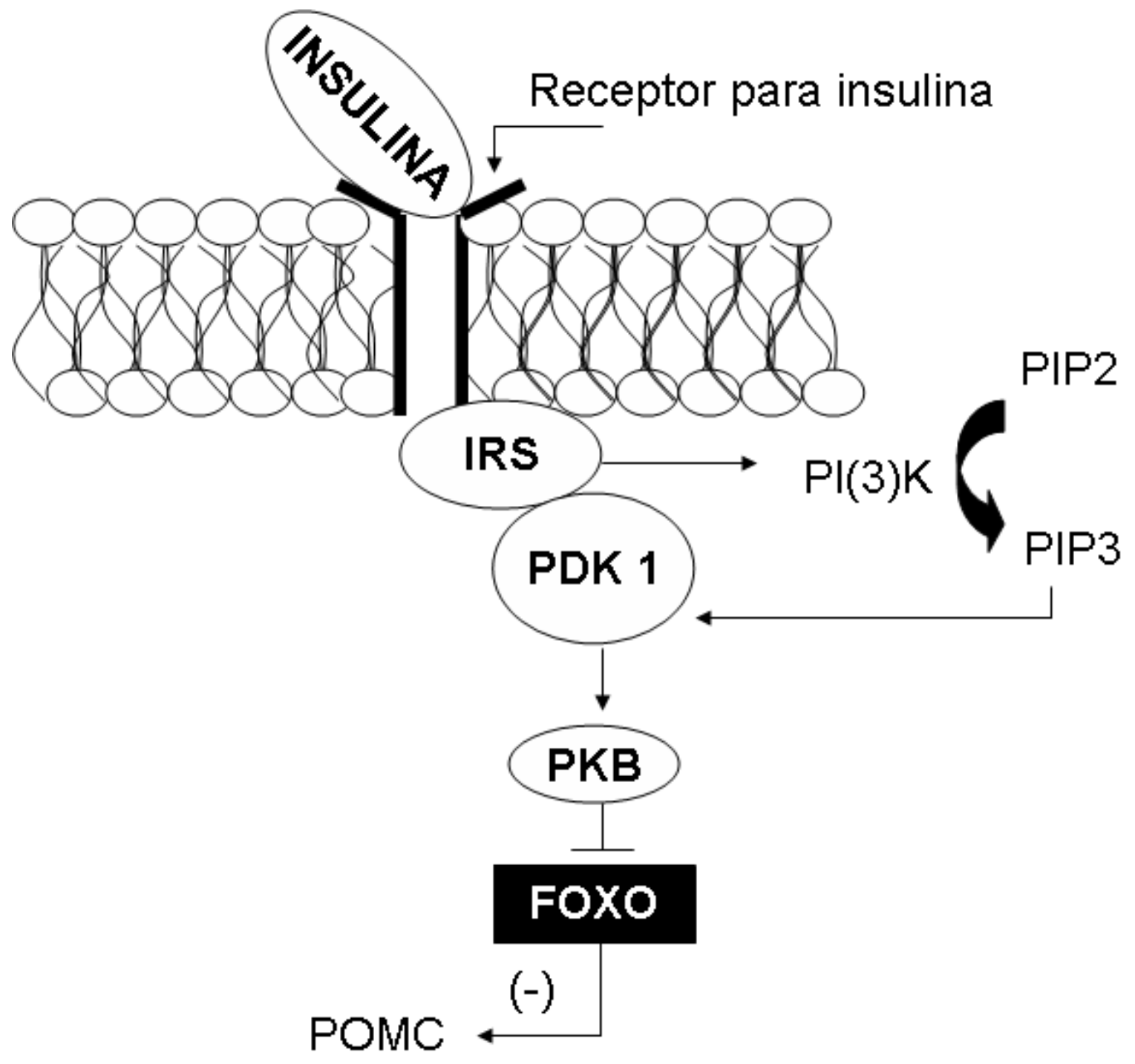

Figura 2. Diagrama esquemático do neurocircuito hipotalâmico representa a ligação da insulina ao seu receptor. Neste esquema inicial ocorre a síntese da enzima fosfatidilinositol-3-OH Kinase $(\mathrm{PI}(3) \mathrm{K})$ a partir da fosforilação do IRS que aumenta a atividade do fosfatidilinositol3,4,5-trifosfato (PIP3). A síntese da PIP3 ativa a proteína kinase dependente de fosfoinositol-3 (PDK1) que expressa à proteína kinase $B$ (PKB) e inibe o fator de transcrição FOXO1 e a atividade da POMC. 
leptina na interação hipotalâmica em modelos animais submetidos à restrição alimentar. ${ }^{37}$ Esta interação ocorre por intermédio de duas vias. $\mathrm{Na} 1^{\circ}$ via tanto a leptina quanto a insulina são responsáveis pela atividade JAK-2 e pela fosforilação da STAT-3, mas a via JAK-2/STAT-3 é controlada diretamente pela leptina, sendo apenas modulada pela ação da insulina. ${ }^{35,38} \mathrm{Na}$ $2^{\circ}$ via ocorre a interação destes dois hormônios na sinalização da via IRS/ PI(3)K, onde a insulina apresenta o papel de sintetiza a PI(3)K e a leptina potencializa o efeito anorexígeno. ${ }^{35,38}$

\section{Conclusão}

No presente trabalho foi descrito inúmeras ações hormonais com interações entre receptores específicos e aumento da expressão de neurônios específicos, tanto orexígenos como anorexígenos, na regulação da ingestão alimentar e no controle do peso corporal. Este fato revela a complexidade do controle do balanço energético e todos os centros envolvidos na busca pela homeostase energética, principalmente, pela interação da insulina, leptina e grelina.

\begin{abstract}
The purpose of a literature review was to explain about the hormonal regulation and neural control of the food intake and body weight. The ingestion of macronutrients, the energy cost of food and hormone synthesis that increases the activity of the orexygenic and anorexygenic neurons are determinative factors in the regulation of the food intake and body weight. The leptin, insulin and peptide YY increase activity of the anorexygenic neurons POMC/CART. However, the ghrelin increases activity of the orexygenic neurons NPY/AgRP. These hormones crossing the barrier hematoencephalic and binds to specifics receptors in the hypothalamic arcuate nucleus. These mechanisms show to the complexity of the food intake control and all the involved centers in the search for the energy homeostasis.
\end{abstract}

Keywords: Hormones. Orexigen and Anorexigen Neurons. Food Intake. Neurons/classification.

\section{Referências Bibliográficas}

1. Stanley S, Wynne K, McGowan B, Bloom S. Hormonal Regulation of Food Intake. Physiol Rev. 2005; 85: 1131-58.

2. Geliebter A. Gastric and capacity in relation to food intake in humans. Physiol Behav 1988;44:665-8.

3. Friedman JM, Halaas JL. Leptin and the regulation of body weight in mammals. Nature. 1998;395:763-70.

4. Schwartz MV. Staying slim with insulin in mind. Science 2000; 289: 2066-7.

5. Woods SC, Seeley RJ, Porte JR. D, Schwartz MW. Signals that regulate food intake and energy homeostasis. Science 1998;280: 1378-83.

6. Berthoud H-R. Homeostatic and nonhomeostatic pathways involved in the control of food intake and energy balance. Obesity. 2006;14(Suppl 5):197S-200S.

7. King BM. The rise, fall, and resurrection of the ventromedial hypothalamus in the regulation of feeding behavior and body weight. Physiol Behav. 2006;87:221-44.

8. Nagamine I, Sunagawa K, Kuriwaki J, Shinjo A. Changes in single unit activity in the lateral hypothalamic area of goats during feeding. J Anim Sci. 2003;81:529-36.

9. Yoshida T, Nishioka H, Nakamura Y, Kondo M. Reduced norepinephrine turnover in mice with monossodium glutamateinduced obesity. Metabolism 1984;33: 1060-3.

10. Grill HJ. Distributed neural control of energy balance: contributions from hindbrain and hypothalamus. Obesity. 2006;14(Suppl 5):216S-21S.
11. Morton GJ, Cummings DE, Baskin DG, Barsh GS, Schwartz MW. Central nervous system control of food intake and body weight. Nature. 2006; 443: 289-95.

12. El Haschimi K, Pierroz DD, Hileman SM, Bjorbaek C, Flier JS. Two defects contribute to hypothalamic leptin resistance in mice with diet-induced obesity. J Clin Invest 2000;105: 182732.

13. Minokoshi Y, Alquier T, Furukawa N, KimYB, Lee A, Xue B, Mu J, Foufelle F, Ferre P, Birnbaum MJ, Stuck BJ, Kahn BB. AMPkinase regulates food intake by responding to hormonal and nutrient signals in the hypothalamus. Nature. 2004; 428: 56974.

14. Hardie DG, Carling D. The AMP-activated protein kinase: fuel gauge of the mammalian cell? Eur J Biochem. 1997;246: 25973.

15. Frühbeck G, Gómez-Ambrosi J, Muruzábal FJ, Burrell MA. The adipocyte: a model for integration of endocrine and metabolic signaling in energy metabolism regulation. Am J Physiol Endocrinol Metab2001;280: 827-47.

16. Date $Y$, Kojima M, Hosoda H, Sawaguchi A, Mondal MS, Suganuma T, Matsukura S, Kangawa K, and Nakazato M. Ghrelin, a novel growth hormone-releasing acylated peptide, is synthesized in a distinct endocrine cell type in the gastrointestinal tracts of rats and humans. Endocrinology. 2000;141: 4255-61.

17. Kojima M, Hosoda H, Date Y, Nakazato M, Matsuo H, Kangawa K. Ghrelin is a growth-hormone-releasing acylated peptide from stomach. Nature. 1999;402:656-60. 
18. Tschop M, Smiley DL, Heiman ML. Ghrelin induces adiposity in rodents. Nature. 2000; 407: 908. 13.

19. Erdmann J, Topsch R, Lippl F, Gussmann P, Schusdziarra V. Postprandial response of plasma ghrelin levels to various test meals in relation to food intake, plasma insulin, and glucose. J Clin Endocrinol Metabol. 2004; 89:3048-54.

20. Date Y, Murakami N, Toshinai K, Matsukura S, Niijima A, Matsuo $\mathrm{H}$, Kangawa $\mathrm{K}$, and Nakazato $\mathrm{M}$. The role of the gastric afferent vagal nerve in ghrelin-induced feeding and growth hormone secretion in rats. Gastroenterology. 2002;123: 11208.

21. Cowley MA, Smith RG, Diano S, Tschop M, Pronchuk N, Grove $\mathrm{KL}$, Strasburger CJ, Bidlingmaier M, Esterman M, Heiman ML, Garcia-Segura LM, Nillni EA, Mendez P, Low MJ, Sotonyi P, Friedman JM, Liu H, Pinto S, Colmers WF, Cone RD, and Horvath TL. The distribution and mechanism of action of ghrelin in the CNS demonstrates a novel hypothalamic circuit regulating energy homeostasis. Neuron 2003;37: 649-61.

22. Toshinai K, Date $Y$, Murakami N, Shimada M, Mondal MS, Shimbara T, Guan JL, Wang QP, Funahashi H, Sakurai T, Shioda S, Matsukura S, Kangawa K, and Nakazato M. Ghrelin induced food intake is mediated via the orexin pathway. Endocrinology. 2003;144: 1506-12.

23. Adrian TE, Ferri GL, Bacarese-Hamilton AJ, FuessI HS, Polak $\mathrm{JM}$, and Bloom SR. Human distribution and release of a putative new gut hormone, peptide YY. Gastroenterology. 1985;89: 1070-7.

24. Konturek SJ, Konturek JW, Pawlik T, Brzozowki T. Brain gut axis and its role in the control of food intake. J Physiol Pharmacol. 2004; 55: 137-54.

25. Batterham RL, Cowley MA, Small CJ, Herzog H, Cohen MA, Dakin CL, Wren AM, Brynes AE, Low MJ, Ghatei MA, Cone RD, Bloom SR. Gut hormone PYY(3-36) physiologically inhibits food intake. Nature. 2002;418: 650-54.

26. Lin HC and Chey WY. Cholecystokinin and peptide YY are released by fat in either proximal or distal small intestine in dogs. Regul Pept. 2003;114: 131-5.
27. Batterham RL, Cohen MA, Ellis SM, Le Roux CW, Wihters DJ, Frost GS, et al. Inhibition of food intake in obese subjects by peptide YY. N Engl J Med. 2003; 349:941-8.

28. Adrian TE, Savage AP, Bacarese-Hamilton AJ, Wolfe K, Besterman AS, Bloom SR. Peptide YY abnormalities in gastrointestinal diseases. Gastroenterology..1986;90:379-84.

29. Porte D Jr, Baskin DG, Schwartz MW. Leptin and insulin action in the central nervous system. Nutr Rev. 2002;60: 20-9.

30. Lam TK, Yoshii H, Haber CA, Bogdanovic E, Lam L, Fantus IG, Giacca A. Free fattyacid-induced hepatic insulin resistance: a potencial role for protein kinase C-d. Am J Physiol Endocrinol Metab. 2002;283:.682-91.

31. Kahn CR, Goldfine AB. Moleculardeterminants of insulin action. J Diabet Complicat.1993;7: 92-105.

32. Van der Heide, LP, Ramakers, GMJ, Smidt MP. Insulin signalling in the central nervous system: learning to survive. Prog Neurobiol. 2006;79: 205-21.

33. Air EL, Strowski MZ, Benoit SC, Conarello SL, Salituro GM, Guan XM, Liu K, Woods SC, and Zhang BB. Small moleculeinsulin mimetics reduce food intake and body weight and prevent development of obesity. Nat Med. 2002;8: 17983.

34. Marks JL, Porte D Jr, Stahl WL, and Baskin DG. Localization of insulin receptor mRNA in rat brain by in situ hybridization. Endocrinology. 1990;127: 3234-6.

35. Seino S, Bell GI. Alternative splicing of human insulin receptor messenger RNA. Biochem Biophys Res Commun. 1989;159: 312-6. Taniguchi CM, Emanuelli B, Kahn CR. Critical nodes in signaling pathways: insights into insulin action. Nature Rev Mol Cell Biol. 2006; 7: 85-96.

36. Air E, Benoit SC, Clegg DJ, Seeley RJ, Woods SC. Insulin and Leptin combine additively to reduce food intake and body weight in rats. Endocrinology. 2002;143:2449-52.

37. Bruning JC, Gautam D, Burks DJ, Gillette J, Schubert M, Orban PC, Klein R, Krone W, Muller-Wieland D, and Kahn CR. Role of brain insulin receptor in control of body weight and reproduction. Science. 2000; 289: 2122-5. 